\title{
Iterative algorithms for a system of generalized variational inequalities in Hilbert spaces
}

Mingliang Zhang*

\section{"Correspondence:}

hdzhangml@yeah.net

School of Mathematics and

Information Science, Henan

University, Kaifeng, 475000, China

\begin{abstract}
In this paper, a new system of generalized nonlinear variational inequalities involving three operators is introduced. A three-step iterative algorithm is considered for the system of generalized nonlinear variational inequalities. Strong convergence theorems of the three-step iterative algorithm are established.
\end{abstract}

MSC: $47 \mathrm{H} 05 ; 47 \mathrm{H} 09 ; 47 \mathrm{~J} 25$

Keywords: variational inequality; projection method; relaxed cocoercive mapping; convergence

\section{Introduction}

Variational inequalities are among the most interesting and intensively studied classes of mathematical problems and have wide applications in the fields of optimization and control, economics, transportation equilibrium and engineering sciences. There exists a vast amount of literature (see, for instance, [1-26]) on the approximation solvability of nonlinear variational inequalities as well as operator equations.

Iterative algorithms have played a central role in the approximation solvability, especially of nonlinear variational inequalities as well as of nonlinear equations, in several fields such as applied mathematics, mathematical programming, mathematical finance, control theory and optimization, engineering sciences and others. Projection methods have played a significant role in the numerical resolution of variational inequalities based on their convergence analysis. However, the convergence analysis does require some sort of strong monotonicity besides the Lipschitz continuity. There have been some recent developments where convergence analysis for projection methods under somewhat weaker conditions such as cocoercivity [28] and partial relaxed monotonicity [24] is achieved.

Recently, Chang et al. [17] introduced a two-step iterative algorithm for a system of nonlinear variational inequalities and established strong convergence theorems. Huang and Noor [16] introduced the so-called explicit two-step iterative algorithm for a system of nonlinear variational inequalities involving two different nonlinear operators and established strong convergence theorems.

In this paper, we consider, based on the projection method, the approximate solvability of a new system of generalized nonlinear variational inequalities involving three different nonlinear operators in the framework of Hilbert spaces. The results presented in this paper

(c) 2012 Zhang; licensee Springer. This is an Open Access article distributed under the terms of the Creative Commons Attribution License (http://creativecommons.org/licenses/by/2.0), which permits unrestricted use, distribution, and reproduction in any medium, provided the original work is properly cited. 
extend and improve the corresponding results announced in Huang and Noor [16], Chang et al. [17], Verma [24-26] and many others.

Let $H$ be a real Hilbert space, whose inner product and norm are denoted by $\langle\cdot, \cdot\rangle$ and $\|\cdot\|$, respectively. Let $C$ be a nonempty closed convex subset of $H$ and $P_{C}$ be the metric projection from $H$ onto $C$.

Given nonlinear operators $T, f: C \rightarrow H$ and $g: C \rightarrow C$, we consider the problem of finding $u \in C$ such that

$$
\langle g(u)-f(u)+\lambda T u, v-g(u)| \geq 0, \quad \forall v \in C,
$$

where $\lambda>0$ is a constant. The variational inequality (1.1) is called the generalized variational inequality involving three operators.

We see that an element $u \in C$ is a solution to the generalized variational inequality (1.1) if and only if $u \in C$ is a fixed point of the mapping

$$
I-g+P_{C}(f-\lambda T)
$$

where $I$ is the identity mapping. This equivalence plays an important role in this work.

If $f=g$, then the generalized variational inequality (1.1) is equivalent to the following.

Find $u \in C$ such that

$$
\langle T u, v-g(u)\rangle \geq 0, \quad \forall v \in C .
$$

Further, if $g=I$, then the problem (1.2) is reduced to finding $u \in C$ such that

$$
\langle T u, v-u\rangle \geq 0, \quad \forall v \in C,
$$

which is known as the classical variational inequality originally introduced and studied by Stampacchia [27].

Let $T: C \rightarrow H$ be a mapping. Recall the following definitions.

(1) $T$ is said to be monotone if

$$
\langle T u-T v, u-v\rangle \geq 0, \quad \forall u, v \in C .
$$

(2) $T$ is called $\delta$-strongly monotone if there exists a constant $\delta>0$ such that

$$
\langle T x-T y, x-y\rangle \geq \delta\|x-y\|^{2}, \quad \forall x, y \in C .
$$

This implies that

$$
\|T x-T y\| \geq \delta\|x-y\|, \quad \forall x, y \in C,
$$

that is, $T$ is $\delta$-expansive.

(3) $T$ is said to be $\gamma$-cocoercive if there exists a constant $\gamma>0$ such that

$$
\langle T x-T y, x-y\rangle \geq \gamma\|T x-T y\|^{2}, \quad \forall x, y \in C .
$$

Clearly, every $\gamma$-cocoercive mapping $A$ is $\frac{1}{\gamma}$-Lipschitz continuous. 
(4) $T$ is said to be relaxed $\gamma$-cocoercive if there exists a constant $\gamma>0$ such that

$$
\langle T x-T y, x-y\rangle \geq(-\gamma)\|T x-T y\|^{2}, \quad \forall x, y \in C .
$$

(5) $T$ is said to be relaxed $(\gamma, \delta)$-cocoercive if there exist two constants $\gamma, \delta>0$ such that

$$
\langle T x-T y, x-y\rangle \geq(-\gamma)\|T x-T y\|^{2}+\delta\|x-y\|^{2}, \quad \forall x, y \in C .
$$

Let $T_{i}: C \times C \times C \rightarrow H, f_{i}: C \rightarrow H$ and $g_{i}: C \rightarrow C$ be nonlinear mappings for each $i=1,2,3$. Consider a system of generalized nonlinear variational inequality (SGNVI) as follows.

Find $\left(x^{*}, y^{*}, z^{*}\right) \in C \times C \times C$ such that for all $s, t, r>0$,

$$
\begin{cases}\left\langle s T_{1}\left(y^{*}, z^{*}, x^{*}\right)+g_{1}\left(x^{*}\right)-f_{1}\left(y^{*}\right), x-g_{1}\left(x^{*}\right)\right\rangle \geq 0, & \forall x \in C, \\ \left\langle t T_{2}\left(z^{*}, x^{*}, y^{*}\right)+g_{2}\left(y^{*}\right)-f_{2}\left(z^{*}\right), x-g_{2}\left(x^{*}\right)\right\rangle \geq 0, & \forall x \in C, \\ \left\langle r T_{3}\left(x^{*}, y^{*}, z^{*}\right)+g_{3}\left(z^{*}\right)-f_{3}\left(x^{*}\right), x-g_{3}\left(x^{*}\right)\right\rangle \geq 0, & \forall x \in C .\end{cases}
$$

One can easily see SGNVI (1.4) is equivalent to the following projection problem:

$$
\begin{cases}g_{1}\left(x^{*}\right)=P_{C}\left(f_{1}\left(y^{*}\right)-s T_{1}\left(y^{*}, z^{*}, x^{*}\right)\right), & \forall s>0, \\ g_{2}\left(y^{*}\right)=P_{C}\left(f_{2}\left(z^{*}\right)-t T_{2}\left(z^{*}, x^{*}, y^{*}\right)\right), & \forall t>0, \\ g_{3}\left(z^{*}\right)=P_{C}\left(f_{3}\left(x^{*}\right)-r T_{3}\left(x^{*}, y^{*}, z^{*}\right)\right), & \forall r>0 .\end{cases}
$$

Next, we consider some special classes of SGNVI (1.4) as follows.

(I) If $g_{1}=g_{2}=g_{3}=I$, then SGNVI (1.4) is reduced to the following.

Find $\left(x^{*}, y^{*}, z^{*}\right) \in C \times C \times C$ such that for all $s, t, r>0$,

$$
\begin{cases}\left\langle s T_{1}\left(y^{*}, z^{*}, x^{*}\right)+x^{*}-f_{1}\left(y^{*}\right), x-x^{*}\right\rangle \geq 0, & \forall x \in C, \\ \left\langle t T_{2}\left(z^{*}, x^{*}, y^{*}\right)+y^{*}-f_{2}\left(z^{*}\right), x-x^{*}\right\rangle \geq 0, & \forall x \in C, \\ \left\langle r T_{3}\left(x^{*}, y^{*}, z^{*}\right)+z^{*}-f_{3}\left(x^{*}\right), x-x^{*}\right\rangle \geq 0, & \forall x \in C .\end{cases}
$$

We see that the problem (1.6) is equivalent to the following projection problem:

$$
\begin{cases}x^{*}=P_{C}\left(f_{1}\left(y^{*}\right)-s T_{1}\left(y^{*}, z^{*}, x^{*}\right)\right), & \forall s>0, \\ y^{*}=P_{C}\left(f_{2}\left(z^{*}\right)-t T_{2}\left(z^{*}, x^{*}, y^{*}\right)\right), & \quad \forall t>0, \\ z^{*}=P_{C}\left(f_{3}\left(x^{*}\right)-r T_{3}\left(x^{*}, y^{*}, z^{*}\right)\right), & \forall r>0 .\end{cases}
$$

(II) If $f_{1}=f_{2}=f_{3}=I$, then SGNVI (1.4) is reduced to the following.

Find $\left(x^{*}, y^{*}, z^{*}\right) \in C \times C \times C$ such that for all $s, t, r>0$,

$$
\begin{cases}\left\langle s T_{1}\left(y^{*}, z^{*}, x^{*}\right)+g_{1}\left(x^{*}\right)-y^{*}, x-g_{1}\left(x^{*}\right)\right\rangle \geq 0, & \forall x \in C, \\ \left\langle t T_{2}\left(z^{*}, x^{*}, y^{*}\right)+g_{2}\left(y^{*}\right)-z^{*}, x-g_{2}\left(x^{*}\right)\right\rangle \geq 0, & \forall x \in C, \\ \left\langle r T_{3}\left(x^{*}, y^{*}, z^{*}\right)+g_{3}\left(z^{*}\right)-x^{*}, x-g_{3}\left(x^{*}\right)\right\rangle \geq 0, & \forall x \in C .\end{cases}
$$


We see that the problem (1.8) is equivalent to the following projection problem:

$$
\begin{cases}g_{1}\left(x^{*}\right)=P_{C}\left[y^{*}-s T_{1}\left(y^{*}, z^{*}, x^{*}\right)\right], & \forall s>0, \\ g_{2}\left(y^{*}\right)=P_{C}\left[z^{*}-t T_{2}\left(z^{*}, x^{*}, y^{*}\right)\right], & \forall t>0, \\ g_{3}\left(z^{*}\right)=P_{C}\left[x^{*}-r T_{3}\left(x^{*}, y^{*}, z^{*}\right)\right], & \forall r>0 .\end{cases}
$$

(III) If $g_{1}=g_{2}=g_{3}=f_{1}=f_{2}=f_{3}=I$, then SGNVI (1.4) is reduced to the following. Find $\left(x^{*}, y^{*}, z^{*}\right) \in C \times C \times C$ such that for all $s, t, r>0$,

$$
\begin{cases}\left\langle s T_{1}\left(y^{*}, z^{*}, x^{*}\right)+x^{*}-y^{*}, x-x^{*}\right\rangle \geq 0, & \forall x \in C, \\ \left\langle t T_{2}\left(z^{*}, x^{*}, y^{*}\right)+y^{*}-z^{*}, x-x^{*}\right\rangle \geq 0, & \forall x \in C, \\ \left\langle r T_{3}\left(x^{*}, y^{*}, z^{*}\right)+z^{*}-x^{*}, x-x^{*}\right\rangle \geq 0, & \forall x \in C .\end{cases}
$$

One can easily get that the problem (1.10) is equivalent to the following projection problem:

$$
\begin{cases}x^{*}=P_{C}\left(y^{*}-s T_{1}\left(y^{*}, z^{*}, x^{*}\right)\right), & \forall s>0, \\ y^{*}=P_{C}\left(z^{*}-t T_{2}\left(z^{*}, x^{*}, y^{*}\right)\right), & \forall t>0, \\ z^{*}=P_{C}\left(x^{*}-r T_{3}\left(x^{*}, y^{*}, z^{*}\right)\right), & \forall r>0 .\end{cases}
$$

(IV) If $T_{1}, T_{2}$ and $T_{3}$ are univariate mappings, then SGNVI (1.4) is reduced to the following.

Find $\left(x^{*}, y^{*}, z^{*}\right) \in C \times C \times C$ such that for all $s, t, r>0$,

$$
\begin{cases}\left\langle s T_{1} y^{*}+g_{1}\left(x^{*}\right)-f_{1}\left(y^{*}\right), x-g_{1}\left(x^{*}\right)\right\rangle \geq 0, & \forall x \in C, \\ \left\langle t T_{2} z^{*}+g_{2}\left(y^{*}\right)-f_{2}\left(z^{*}\right), x-g_{2}\left(x^{*}\right)\right\rangle \geq 0, & \forall x \in C, \\ \left\langle r T_{3} x^{*}+g_{3}\left(z^{*}\right)-f_{3}\left(x^{*}\right), x-g_{3}\left(x^{*}\right)\right\rangle \geq 0, & \forall x \in C .\end{cases}
$$

One can easily see that the problem (1.12) is equivalent to the following projection problem:

$$
\begin{cases}g_{1}\left(x^{*}\right)=P_{C}\left(f_{1}\left(y^{*}\right)-s T_{1} y^{*}\right), & \forall s>0, \\ g_{2}\left(y^{*}\right)=P_{C}\left(f_{2}\left(z^{*}\right)-t T_{2} z^{*}\right), & \forall t>0, \\ g_{3}\left(z^{*}\right)=P_{C}\left(f_{3}\left(x^{*}\right)-r T_{3} x^{*}\right), & \forall r>0 .\end{cases}
$$

\section{Preliminaries}

In this section, to study the approximate solvability of the problems (1.4), (1.6), (1.8), (1.10) and (1.12), we introduce the following three-step algorithms.

Algorithm 2.1 For any $\left(x_{0}, y_{0}, z_{0}\right) \in C \times C \times C$, compute the sequences $\left\{x_{n}\right\},\left\{y_{n}\right\}$ and $\left\{z_{n}\right\}$ by the following iterative process:

$$
\begin{cases}x_{n+1}=\left(1-\alpha_{n}\right) x_{n}+\alpha_{n}\left(x_{n}-g_{1}\left(x_{n}\right)+P_{C}\left(f_{1}\left(y_{n}\right)-s T_{1}\left(y_{n}, z_{n}, x_{n}\right)\right)\right), & n \geq 0 \\ g_{3}\left(z_{n+1}\right)=P_{C}\left(f_{3}\left(x_{n+1}\right)-r T_{3}\left(x_{n+1}, y_{n}, z_{n}\right)\right), & n \geq 0 \\ g_{2}\left(y_{n+1}\right)=P_{C}\left(f_{2}\left(z_{n+1}\right)-t T_{2}\left(z_{n+1}, x_{n}, y_{n}\right)\right), & n \geq 0\end{cases}
$$

where $r, s, t>0$ are three constants and $\left\{\alpha_{n}\right\}$ is a sequence in $[0,1]$. 
If $g_{1}=g_{2}=g_{3}=I$, then Algorithm 2.1 is reduced to the following.

Algorithm 2.2 For any $\left(x_{0}, y_{0}, z_{0}\right) \in C \times C \times C$, compute the sequences $\left\{x_{n}\right\},\left\{y_{n}\right\}$ and $\left\{z_{n}\right\}$ by the following iterative process:

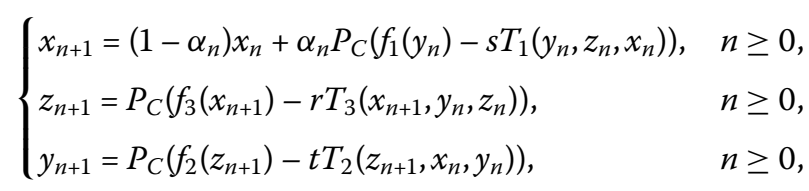

where $r, s, t>0$ are three constants and $\left\{\alpha_{n}\right\}$ is a sequence in $[0,1]$.

If $f_{1}=f_{2}=f_{3}=I$, the identity mapping, then Algorithm 2.1 is reduced to the following.

Algorithm 2.3 For any $\left(x_{0}, y_{0}, z_{0}\right) \in C \times C \times C$, compute the sequences $\left\{x_{n}\right\},\left\{y_{n}\right\}$ and $\left\{z_{n}\right\}$ by the following iterative process:

$$
\begin{cases}x_{n+1}=\left(1-\alpha_{n}\right) x_{n}+\alpha_{n}\left(x_{n}-g_{1}\left(x_{n}\right)+P_{C}\left(y_{n}-s T_{1}\left(y_{n}, z_{n}, x_{n}\right)\right)\right), & n \geq 0 \\ g_{3}\left(z_{n+1}\right)=P_{C}\left(x_{n+1}-r T_{3}\left(x_{n+1}, y_{n}, z_{n}\right)\right), & n \geq 0 \\ g_{2}\left(y_{n+1}\right)=P_{C}\left(z_{n+1}-t T_{2}\left(z_{n+1}, x_{n}, y_{n}\right)\right), & n \geq 0\end{cases}
$$

where $r, s, t>0$ are three constants and $\left\{\alpha_{n}\right\}$ is a sequence in $[0,1]$.

If $f_{1}=f_{2}=f_{3}=g_{1}=g_{2}=g_{3}=I$, the identity mapping, then Algorithm 2.1 is reduced to the following.

Algorithm 2.4 For any $\left(x_{0}, y_{0}, z_{0}\right) \in C \times C \times C$, compute the sequences $\left\{x_{n}\right\},\left\{y_{n}\right\}$ and $\left\{z_{n}\right\}$ by the following iterative process:

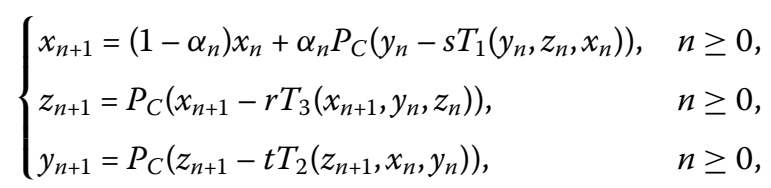

where $r, s, t>0$ are three constants and $\left\{\alpha_{n}\right\}$ is a sequence in $[0,1]$.

(IV) If $T_{1}, T_{2}$ and $T_{3}$ are univariate mappings, then Algorithm 2.1 is reduced to the following.

Algorithm 2.5 For any $\left(x_{0}, y_{0}, z_{0}\right) \in C \times C \times C$, compute the sequences $\left\{x_{n}\right\},\left\{y_{n}\right\}$ and $\left\{z_{n}\right\}$ by the following iterative process:

$$
\begin{cases}x_{n+1}=\left(1-\alpha_{n}\right) x_{n}+\alpha_{n}\left(x_{n}-g_{1}\left(x_{n}\right)+P_{C}\left(f_{1}\left(y_{n}\right)-s T_{1} y_{n}\right)\right), & n \geq 0, \\ g_{3}\left(z_{n+1}\right)=P_{C}\left(f_{3}\left(x_{n+1}\right)-r T_{3} x_{n+1}\right), & n \geq 0, \\ g_{2}\left(y_{n+1}\right)=P_{C}\left(f_{2}\left(z_{n+1}\right)-t T_{2} z_{n+1}\right), & n \geq 0,\end{cases}
$$

where $r, s, t>0$ are three constants and $\left\{\alpha_{n}\right\}$ is a sequence in $[0,1]$.

In order to prove our main results, we also need the following lemma and definitions. 
Lemma 2.6 [29] Assume that $\left\{a_{n}\right\}$ is a sequence of nonnegative real numbers such that

$$
a_{n+1} \leq\left(1-\lambda_{n}\right) a_{n}+b_{n}, \quad \forall n \geq n_{0}
$$

where $n_{0}$ is a nonnegative integer, $\left\{\lambda_{n}\right\}$ is a sequence in $(0,1)$ with $\sum_{n=1}^{\infty} \lambda_{n}=\infty$ and $b_{n}=$ $\circ\left(\lambda_{n}\right)$, then $\lim _{n \rightarrow \infty} a_{n}=0$.

Definition 2.7 A mapping $T: C \times C \times C \rightarrow H$ is said to be relaxed $(\gamma, \delta)$-cocoercive if there exist constants $\gamma, \delta>0$ such that for all $x, x^{\prime} \in C$,

$$
\begin{aligned}
& \left\langle T(x, y, z)-T\left(x^{\prime}, y^{\prime}, z^{\prime}\right), x-x^{\prime}\right\rangle \\
& \quad \geq(-\gamma)\left\|T(x, y, z)-T\left(x^{\prime}, y^{\prime}, z^{\prime}\right)\right\|^{2}+\delta\left\|x-x^{\prime}\right\|^{2}, \quad \forall y, y^{\prime}, z, z^{\prime} \in C .
\end{aligned}
$$

Definition 2.8 A mapping $T: C \times C \times C \rightarrow H$ is said to be $\beta$-Lipschitz continuous in the first variable if there exists a constant $\beta>0$ such that for all $x, x^{\prime} \in C$,

$$
\left\|T(x, y, z)-T\left(x^{\prime}, y^{\prime}, z^{\prime}\right)\right\| \leq \beta\left\|x-x^{\prime}\right\|, \quad \forall y, y^{\prime}, z, z^{\prime} \in C .
$$

\section{Main results}

Theorem 3.1 Let $C$ be a nonempty, closed and convex subset of a real Hilbert space $H$. Let $T_{i}: C \times C \times C \rightarrow H$ be a relaxed $\left(\gamma_{i}, \delta_{i}\right)$-cocoercive and $\beta_{i}$-Lipschitz continuous mapping in the first variable, $f_{i}: C \rightarrow H$ be a relaxed $\left(\eta_{i}, \rho_{i}\right)$-cocoercive and $\lambda_{i}$-Lipschitz continuous mapping and $g_{i}: C \rightarrow C$ be a relaxed $\left(\bar{\eta}_{i}, \bar{\rho}_{i}\right)$-cocoercive and $\bar{\lambda}_{i}$-Lipschitz continuous mapping for each $i=1,2,3$. Suppose that $\left(x^{*}, y^{*}, z^{*}\right) \in C \times C \times C$ is a solution to the problem (1.4). Let $\left\{x_{n}\right\},\left\{y_{n}\right\}$ and $\left\{z_{n}\right\}$ be the sequences generated by Algorithm 2.1. Assume that the following conditions are satisfied:

(a) $\sum_{n=0}^{\infty} \alpha_{n}=\infty$;

(b) $0 \leq \theta_{6}, \theta_{9}<1$;

(c) $\left(\theta_{1}+\theta_{2}\right)\left(\theta_{4}+\theta_{5}\right)\left(\theta_{7}+\theta_{8}\right) \leq\left(1-\theta_{3}\right)\left(1-\theta_{6}\right)\left(1-\theta_{9}\right)$,

where

$$
\begin{array}{ll}
\theta_{1}=\sqrt{1-2 s \delta_{1}+2 s \gamma_{1} \beta_{1}^{2}+s^{2} \beta_{1}^{2}}, & \theta_{2}=\sqrt{1-2 \rho_{1}+\lambda_{1}^{2}+2 \eta_{1} \lambda_{1}^{2}}, \\
\theta_{3}=\sqrt{1-2 \bar{\rho}_{1}+\bar{\lambda}_{1}^{2}+2 \bar{\eta}_{1} \bar{\lambda}_{1}^{2}}, & \theta_{4}=\sqrt{1-2 t \delta_{2}+2 t \gamma_{2} \beta_{2}^{2}+t^{2} \beta_{2}^{2}}, \\
\theta_{5}=\sqrt{1-2 \rho_{2}+\lambda_{2}^{2}+2 \eta_{2} \lambda_{2}^{2}}, & \theta_{6}=\sqrt{1-2 \bar{\rho}_{2}+\bar{\lambda}_{2}^{2}+2 \bar{\eta}_{2} \bar{\lambda}_{2}^{2}}, \\
\theta_{7}=\sqrt{1-2 r \delta_{3}+2 r \gamma_{3} \beta_{3}^{2}+r^{2} \beta_{3}^{2}}, & \theta_{8}=\sqrt{1-2 \rho_{3}+\lambda_{3}^{2}+2 \eta_{3} \lambda_{3}^{2}},
\end{array}
$$

and

$$
\theta_{9}=\sqrt{1-2 \bar{\rho}_{3}+\bar{\lambda}_{3}^{2}+2 \bar{\eta}_{3} \bar{\lambda}_{3}^{2}}
$$

Then the sequences $\left\{x_{n}\right\},\left\{y_{n}\right\}$ and $\left\{z_{n}\right\}$ converge strongly to $x^{\prime \prime}, y^{\prime \prime}$ and $z^{*}$, respectively. 
Proof In view of $\left(x^{*}, y^{*}, z^{*}\right)$ being a solution to the problem (1.4), we see that

$$
\begin{cases}x^{*}=\left(1-\alpha_{n}\right) x^{*}+\alpha_{n}\left(x^{*}-g_{1}\left(x^{*}\right)+P_{C}\left(f_{1}\left(y^{*}\right)-s T_{1}\left(y^{*}, z^{*}, x^{*}\right)\right)\right), & n \geq 0, \\ g_{3}\left(z^{*}\right)=P_{C}\left(f_{3}\left(x^{*}\right)-r T_{3}\left(x^{*}, y^{*}, z^{*}\right)\right), & n \geq 0, \\ g_{2}\left(y^{*}\right)=P_{C}\left(f_{2}\left(z^{*}\right)-t T_{2}\left(z^{*}, x^{*}, y^{*}\right)\right), & n \geq 0 .\end{cases}
$$

It follows from Algorithm (2.1) that

$$
\begin{aligned}
&\left\|x_{n+1}-x^{*}\right\| \\
&=\left\|\left(1-\alpha_{n}\right) x_{n}+\alpha_{n}\left(x_{n}-g_{1}\left(x_{n}\right)+P_{C}\left(f_{1}\left(y_{n}\right)-s T_{1}\left(y_{n}, z_{n}, x_{n}\right)\right)\right)-x^{*}\right\| \\
& \leq\left(1-\alpha_{n}\right)\left\|x_{n}-x^{*}\right\|+\alpha_{n} \|\left(x_{n}-g_{1}\left(x_{n}\right)+P_{C}\left(f_{1}\left(y_{n}\right)-s T_{1}\left(y_{n}, z_{n}, x_{n}\right)\right)\right) \\
&-\left(x^{*}-g_{1}\left(x^{*}\right)+P_{C}\left(f_{1}\left(y^{*}\right)-s T_{1}\left(y^{*}, z^{*}, x^{*}\right)\right)\right) \| \\
& \leq\left(1-\alpha_{n}\right)\left\|x_{n}-x^{*}\right\|+\alpha_{n}\left\|x_{n}-x^{*}-\left(g_{1}\left(x_{n}\right)-g_{1}\left(x^{*}\right)\right)\right\|+\alpha_{n} \| f_{1}\left(y_{n}\right)-f_{1}\left(y^{*}\right) \\
&-s\left(T_{1}\left(y_{n}, z_{n}, x_{n}\right)-T_{1}\left(y^{*}, z^{*}, x^{*}\right)\right) \| \\
& \leq\left(1-\alpha_{n}\right)\left\|x_{n}-x^{*}\right\|+\alpha_{n}\left\|x_{n}-x^{*}-\left(g_{1}\left(x_{n}\right)-g_{1}\left(x^{*}\right)\right)\right\| \\
&+\alpha_{n}\left\|y_{n}-y^{*}-\left(f_{1}\left(y_{n}\right)-f_{1}\left(y^{*}\right)\right)\right\| \\
&+\alpha_{n}\left\|\left(y_{n}-y^{*}\right)-s\left(T_{1}\left(y_{n}, z_{n}, x_{n}\right)-T_{1}\left(y^{*}, z^{*}, x^{*}\right)\right)\right\| .
\end{aligned}
$$

By the assumption that $T_{1}$ is relaxed $\left(\gamma_{1}, r_{1}\right)$-cocoercive and $\beta_{1}$-Lipschitz continuous in the first variable, we obtain that

$$
\begin{aligned}
& \left\|\left(y_{n}-y^{*}\right)-s\left(T_{1}\left(y_{n}, z_{n}, x_{n}\right)-T_{1}\left(y^{*}, z^{*}, x^{*}\right)\right)\right\|^{2} \\
& =\left\|y_{n}-y^{\prime \prime}\right\|^{2}-2 s\left(T_{1}\left(y_{n}, z_{n}, x_{n}\right)-T_{1}\left(y^{\prime \prime}, z^{\prime \prime}, x^{\prime \prime}\right), y_{n}-y^{\prime \prime}\right\rangle \\
& +s^{2}\left\|T_{1}\left(y_{n}, z_{n}, x_{n}\right)-T_{1}\left(y^{*}, z^{*}, x^{*}\right)\right\|^{2} \\
& \leq\left\|y_{n}-y^{*}\right\|^{2}-2 s\left(\left(-\gamma_{1}\right)\left\|T_{1}\left(y_{n}, z_{n}, x_{n}\right)-T_{1}\left(y^{*}, z^{*}, x^{*}\right)\right\|^{2}+\delta_{1}\left\|y_{n}-y^{*}\right\|^{2}\right) \\
& +s^{2}\left\|T_{1}\left(y_{n}, z_{n}, x_{n}\right)-T_{1}\left(y^{*}, z^{*}, x^{*}\right)\right\|^{2} \\
& =\left(1-2 s \delta_{1}\right)\left\|y_{n}-y^{*}\right\|^{2}+\left(2 s \gamma_{1}+s^{2}\right)\left\|T_{1}\left(y_{n}, z_{n}, x_{n}\right)-T_{1}\left(y^{*}, z^{\prime \prime}, x^{*}\right)\right\|^{2} \\
& \leq\left(1-2 s \delta_{1}\right)\left\|y_{n}-y^{*}\right\|^{2}+\left(2 s \gamma_{1}+s^{2}\right) \beta_{1}^{2}\left\|y_{n}-y^{*}\right\|^{2} \\
& =\theta_{1}^{2}\left\|y_{n}-y^{*}\right\|^{2} \text {, }
\end{aligned}
$$

where $\theta_{1}=\sqrt{1-2 s \delta_{1}+2 s \gamma_{1} \beta_{1}^{2}+s^{2} \beta_{1}^{2}}$. On the other hand, it follows from the assumption that $f_{1}$ is relaxed $\left(\eta_{1}, \rho_{1}\right)$-cocoercive and $\lambda_{1}$-Lipschitz continuous that

$$
\begin{aligned}
& \left\|y_{n}-y^{*}-\left(f_{1}\left(y_{n}\right)-f_{1}\left(y^{*}\right)\right)\right\|^{2} \\
& \quad=\left\|y_{n}-y^{*}\right\|^{2}-2\left(f_{1}\left(y_{n}\right)-f_{1}\left(y^{*}\right), y_{n}-y^{*}\right\rangle+\left\|f_{1}\left(y_{n}\right)-f_{1}\left(y^{*}\right)\right\|^{2} \\
& \quad \leq\left\|y_{n}-y^{*}\right\|^{2}-2\left(\left(-\eta_{1}\right)\left\|f_{1}\left(y_{n}\right)-f_{1}\left(y^{*}\right)\right\|^{2}+\rho_{1}\left\|y_{n}-y^{*}\right\|^{2}\right)+\lambda_{1}^{2}\left\|y_{n}-y^{*}\right\|^{2} \\
& \quad=\left(1-2 \rho_{1}+\lambda_{1}^{2}\right)\left\|y_{n}-y^{*}\right\|^{2}+2 \eta_{1}\left\|f_{1}\left(y_{n}\right)-f_{1}\left(y^{*}\right)\right\|^{2} \\
& \quad \leq \theta_{2}^{2}\left\|y_{n}-y^{*}\right\|^{2},
\end{aligned}
$$


where $\theta_{2}=\sqrt{1-2 \rho_{1}+\lambda_{1}^{2}+2 \eta_{1} \lambda_{1}^{2}}$. In a similar way, we can obtain that

$$
\left\|x_{n}-x^{*}-\left(g_{1}\left(x_{n}\right)-g_{1}\left(x^{*}\right)\right)\right\| \leq \theta_{3}\left\|x_{n}-x^{*}\right\|
$$

where $\theta_{3}=\sqrt{1-2 \bar{\rho}_{1}+\bar{\lambda}_{1}^{2}+2 \bar{\eta}_{1} \bar{\lambda}_{1}^{2}}$. Substituting (3.2), (3.3) and (3.4) into (3.1), we arrive at

$$
\left\|x_{n+1}-x^{*}\right\| \leq\left[1-\alpha_{n}\left(1-\theta_{3}\right)\right]\left\|x_{n}-x^{*}\right\|+\alpha_{n}\left(\theta_{1}+\theta_{2}\right)\left\|y_{n}-y^{*}\right\| \text {. }
$$

Next, we estimate $\left\|y_{n}-y^{*}\right\|$. From Algorithm 2.1, we see that

$$
\begin{aligned}
\left\|g_{2}\left(y_{n+1}\right)-g_{2}\left(y^{*}\right)\right\|= & \| P_{C}\left(f_{2}\left(z_{n+1}\right)-t T_{2}\left(z_{n+1}, x_{n}, y_{n}\right)\right) \\
& -P_{C}\left(f_{2}\left(z^{*}\right)-t T_{2}\left(z^{*}, x^{*}, y^{*}\right)\right) \| \\
\leq & \left\|\left(f_{2}\left(z_{n+1}\right)-t T_{2}\left(z_{n+1}, x_{n}, y_{n}\right)\right)-\left(f_{2}\left(z^{*}\right)-t T_{2}\left(z^{*}, x^{*}, y^{*}\right)\right)\right\| \\
\leq & \left\|\left(z_{n+1}-z^{*}\right)-\left(f_{2}\left(z_{n+1}\right)-f_{2}\left(z^{*}\right)\right)\right\| \\
& +\left\|\left(z_{n+1}-z^{*}\right)-t\left(T_{2}\left(z_{n+1}, x_{n}, y_{n}\right)-T_{2}\left(z^{*}, x^{*}, y^{*}\right)\right)\right\| .
\end{aligned}
$$

By the assumption that $T_{2}$ is relaxed $\left(\gamma_{2}, r_{2}\right)$-cocoercive and $\beta_{2}$-Lipschitz continuous in the first variable, we obtain that

$$
\begin{aligned}
& \left\|\left(z_{n+1}-z^{*}\right)-t\left(T_{2}\left(z_{n+1}, x_{n}, y_{n}\right)-T_{2}\left(z^{*}, x^{*}, y^{*}\right)\right)\right\|^{2} \\
& =\left\|z_{n+1}-z^{*}\right\|^{2}-2 t\left|T_{2}\left(z_{n+1}, x_{n}, y_{n}\right)-T_{2}\left(z^{*}, x^{*}, y^{*}\right), z_{n+1}-z^{*}\right\rangle \\
& +t^{2}\left\|T_{2}\left(z_{n+1}, x_{n}, y_{n}\right)-T_{2}\left(z^{*}, x^{*}, y^{*}\right)\right\|^{2} \\
& \leq\left\|z_{n+1}-z^{*}\right\|^{2}-2 t\left(\left(-\gamma_{2}\right)\left\|T_{2}\left(z_{n+1}, x_{n}, y_{n}\right)-T_{2}\left(z^{*}, x^{*}, y^{*}\right)\right\|^{2}+\delta_{2}\left\|z_{n+1}-z^{*}\right\|^{2}\right) \\
& +t^{2}\left\|T_{2}\left(z_{n+1}, x_{n}, y_{n}\right)-T_{2}\left(z^{*}, x^{*}, y^{*}\right)\right\|^{2} \\
& =\left(1-2 t \delta_{2}\right)\left\|z_{n+1}-z^{*}\right\|^{2}+\left(2 t \gamma_{2}+t^{2}\right)\left\|T_{2}\left(z_{n+1}, x_{n}, y_{n}\right)-T_{2}\left(z^{*}, x^{*}, y^{*}\right)\right\|^{2} \\
& \leq\left(1-2 t \delta_{2}\right)\left\|z_{n+1}-z^{*}\right\|^{2}+\left(2 t \gamma_{2}+t^{2}\right) \beta_{2}^{2}\left\|z_{n+1}-z^{*}\right\|^{2} \\
& =\theta_{4}^{2}\left\|z_{n+1}-z^{*}\right\|^{2}
\end{aligned}
$$

where $\theta_{4}=\sqrt{1-2 t \delta_{2}+2 t \gamma_{2} \beta_{2}^{2}+t^{2} \beta_{2}^{2}}$. It follows from the assumption that $f_{2}$ is relaxed $\left(\eta_{2}, \rho_{2}\right)$-cocoercive and $\lambda_{2}$-Lipschitz continuous that

$$
\begin{aligned}
& \left\|\left(z_{n+1}-z^{*}\right)-\left(f_{2}\left(z_{n+1}\right)-f_{2}\left(z^{*}\right)\right)\right\|^{2} \\
& \quad=\left\|z_{n+1}-z^{*}\right\|^{2}-2\left(f_{2}\left(z_{n+1}\right)-f_{2}\left(z^{*}\right), z_{n}-z^{*}\right\rangle+\left\|f_{2}\left(z_{n+1}\right)-f_{2}\left(z^{*}\right)\right\|^{2} \\
& \quad \leq\left\|z_{n+1}-z^{*}\right\|^{2}-2\left(\left(-\eta_{2}\right)\left\|f_{2}\left(z_{n+1}\right)-f_{2}\left(z^{*}\right)\right\|^{2}+\rho_{2}\left\|z_{n+1}-z^{*}\right\|^{2}\right)+\lambda_{2}^{2}\left\|z_{n+1}-z^{*}\right\|^{2} \\
& \quad=\left(1-2 \rho_{2}+\lambda_{2}^{2}\right)\left\|z_{n+1}-z^{*}\right\|^{2}+2 \eta_{2}\left\|f_{2}\left(z_{n+1}\right)-f_{2}\left(z^{*}\right)\right\|^{2} \\
& \quad=\theta_{5}^{2}\left\|z_{n+1}-z^{*}\right\|^{2}
\end{aligned}
$$

where $\theta_{5}=\sqrt{1-2 \rho_{2}+\lambda_{2}^{2}+2 \eta_{2} \lambda_{2}^{2}}$. Substituting (3.7) and (3.8) into (3.6), we see that

$$
\left\|g_{2}\left(y_{n+1}\right)-g_{2}\left(y^{*}\right)\right\| \leq\left(\theta_{4}+\theta_{5}\right)\left\|z_{n+1}-z^{*}\right\| \text {. }
$$


On the other hand, we have

$$
\left\|y_{n+1}-y^{*}\right\| \leq\left\|y_{n+1}-y^{*}-\left(g_{2}\left(y_{n+1}\right)-g_{2}\left(y^{*}\right)\right)\right\|+\left\|g_{2}\left(y_{n+1}\right)-g_{2}\left(y^{*}\right)\right\| .
$$

From the proof of (3.8), we arrive at

$$
\left\|\left(y_{n+1}-y^{\prime \prime}\right)-\left(g_{2}\left(y_{n+1}\right)-g_{2}\left(y^{\prime \prime}\right)\right)\right\| \leq \theta_{6}\left\|y_{n+1}-y^{\prime \prime}\right\|,
$$

where $\theta_{6}=\sqrt{1-2 \bar{\rho}_{2}+\bar{\lambda}_{2}^{2}+2 \bar{\eta}_{2} \bar{\lambda}_{2}^{2}}$. Substituting (3.9) and (3.11) into (3.10), we see that

$$
\left\|y_{n+1}-y^{*}\right\| \leq \theta_{6}\left\|y_{n+1}-y^{*}\right\|+\left(\theta_{4}+\theta_{5}\right)\left\|z_{n+1}-z^{*}\right\| .
$$

It follows from the condition (b) that

$$
\left\|y_{n+1}-y^{*}\right\| \leq \frac{\theta_{4}+\theta_{5}}{1-\theta_{6}}\left\|z_{n+1}-z^{*}\right\| .
$$

That is,

$$
\left\|y_{n}-y^{*}\right\| \leq \frac{\theta_{4}+\theta_{5}}{1-\theta_{6}}\left\|z_{n}-z^{*}\right\|
$$

Finally, we estimate $\left\|z_{n}-z^{*}\right\|$. It follows from Algorithm 2.1 that

$$
\begin{aligned}
& \left\|g_{3}\left(z_{n+1}\right)-g_{3}\left(z^{*}\right)\right\| \\
& =\left\|P_{C}\left(f_{3}\left(x_{n+1}\right)-r T_{3}\left(x_{n+1}, y_{n}, z_{n}\right)\right)-P_{C}\left[f_{3}\left(x^{*}\right)-r T_{3}\left(x^{*}, y^{*}, z^{*}\right)\right]\right\| \\
& \leq\left\|\left(x_{n+1}-x^{*}\right)-\left(f_{3}\left(x_{n+1}\right)-f\left(x^{*}\right)\right)\right\| \\
& \quad+\left\|\left(x_{n+1}-x^{*}\right)-r\left(T_{3}\left(x_{n+1}, y_{n}, z_{n}\right)-T_{3}\left(x^{* \prime}, y^{*}, z^{*}\right)\right)\right\| .
\end{aligned}
$$

In a similar way, we can show that

$$
\left\|\left(x_{n+1}-x^{*}\right)-r\left(T_{3}\left(x_{n+1}, y_{n}, z_{n}\right)-T_{3}\left(x^{*}, y^{*}, z^{*}\right)\right)\right\| \leq \theta_{7}\left\|x_{n+1}-x^{*}\right\|
$$

and

$$
\left\|\left(x_{n+1}-x^{*}\right)-\left(f_{3}\left(x_{n+1}\right)-f_{3}\left(x^{*}\right)\right)\right\| \leq \theta_{8}\left\|x_{n+1}-x^{*}\right\|,
$$

where $\theta_{7}=\sqrt{1-2 r \delta_{3}+2 r \gamma_{3} \beta_{3}^{2}+r^{2} \beta_{3}^{2}}$ and $\theta_{8}=\sqrt{1-2 \rho_{3}+\lambda_{3}^{2}+2 \eta_{3} \lambda_{3}^{2}}$. Substituting (3.14) and (3.15) into (3.13), we arrive at

$$
\left\|g_{3}\left(z_{n+1}\right)-g_{3}\left(z^{*}\right)\right\| \leq\left(\theta_{7}+\theta_{8}\right)\left\|x_{n+1}-x^{*}\right\| .
$$

Note that

$$
\left\|z_{n+1}-z^{*}\right\| \leq\left\|z_{n+1}-z^{\prime \prime}-\left(g_{3}\left(z_{n+1}\right)-g_{3}\left(z^{\prime \prime}\right)\right)\right\|+\left\|g_{3}\left(z_{n+1}\right)-g_{3}\left(z^{\prime \prime}\right)\right\| .
$$


On the other hand, we have

$$
\left\|z_{n+1}-z^{*}-\left(g_{3}\left(z_{n+1}\right)-g_{3}\left(z^{*}\right)\right)\right\| \leq \theta_{9}\left\|z_{n+1}-z^{*}\right\|
$$

$\theta_{9}=\sqrt{1-2 \bar{\rho}_{3}+\bar{\lambda}_{3}^{2}+2 \bar{\eta}_{3} \bar{\lambda}_{3}^{2}}$. Substituting (3.16) and (3.18) into (3.17), we arrive at

$$
\left\|z_{n+1}-z^{*}\right\| \leq \theta_{9}\left\|z_{n+1}-z^{*}\right\|+\left(\theta_{7}+\theta_{8}\right)\left\|x_{n+1}-x^{*}\right\| .
$$

It follows from the condition (b) that

$$
\left\|z_{n+1}-z^{*}\right\| \leq \frac{\theta_{7}+\theta_{8}}{1-\theta_{9}}\left\|x_{n+1}-x^{*}\right\| .
$$

That is,

$$
\left\|z_{n}-z^{*}\right\| \leq \frac{\theta_{7}+\theta_{8}}{1-\theta_{9}}\left\|x_{n}-x^{*}\right\|
$$

Combining (3.5), (3.12) with (3.19), we obtain that

$$
\left\|x_{n+1}-x^{* \prime}\right\| \leq\left(1-\alpha_{n}\left(1-\theta_{3}-\left(\theta_{1}+\theta_{2}\right) \frac{\theta_{4}+\theta_{5}}{1-\theta_{6}} \frac{\theta_{7}+\theta_{8}}{1-\theta_{9}}\right)\right)\left\|x_{n}-x^{*}\right\| .
$$

Since $\sum_{n=0}^{\infty} \alpha_{n}=\infty$ and the condition (c), we can conclude the desired conclusion easily from Lemma 2.6. This completes the proof.

Remark 3.2 Theorem 3.1 includes the corresponding results in Huang and Noor [16] Chang et al. [17], and Verma [24-26] as special cases.

From Theorem 3.1, we can get the following results immediately.

Corollary 3.3 Let $C$ be a nonempty, closed and convex subset of a real Hilbert space $H$. Let $T_{i}: C \times C \times C \rightarrow H$ be a relaxed $\left(\gamma_{i}, \delta_{i}\right)$-cocoercive and $\beta_{i}$-Lipschitz continuous mapping in the first variable and $f_{i}: C \rightarrow H$ be a relaxed $\left(\eta_{i}, \rho_{i}\right)$-cocoercive and $\lambda_{i}$-Lipschitz continuous mapping for each $i=1,2,3$. Suppose that $\left(x^{*}, y^{*}, z^{*}\right) \in C \times C \times C$ is a solution to the problem (1.6). Let $\left\{x_{n}\right\},\left\{y_{n}\right\}$ and $\left\{z_{n}\right\}$ be the sequences generated by Algorithm 2.2. Assume that the following conditions are satisfied:

(a) $\sum_{n=0}^{\infty} \alpha_{n}=\infty$;

(b) $\left(\theta_{1}+\theta_{2}\right)\left(\theta_{4}+\theta_{5}\right)\left(\theta_{7}+\theta_{8}\right) \leq 1$,

where

$$
\begin{array}{ll}
\theta_{1}=\sqrt{1-2 s \delta_{1}+2 s \gamma_{1} \beta_{1}^{2}+s^{2} \beta_{1}^{2}}, & \theta_{2}=\sqrt{1-2 \rho_{1}+\lambda_{1}^{2}+2 \eta_{1} \lambda_{1}^{2}}, \\
\theta_{4}=\sqrt{1-2 t \delta_{2}+2 t \gamma_{2} \beta_{2}^{2}+t^{2} \beta_{2}^{2}}, & \theta_{5}=\sqrt{1-2 \rho_{2}+\lambda_{2}^{2}+2 \eta_{2} \lambda_{2}^{2}},
\end{array}
$$

and

$$
\theta_{7}=\sqrt{1-2 r \delta_{3}+2 r \gamma_{3} \beta_{3}^{2}+r^{2} \beta_{3}^{2}}, \quad \theta_{8}=\sqrt{1-2 \rho_{3}+\lambda_{3}^{2}+2 \eta_{3} \lambda_{3}^{2}} .
$$

Then the sequences $\left\{x_{n}\right\},\left\{y_{n}\right\}$ and $\left\{z_{n}\right\}$ converge strongly to $x^{\prime \prime}, y^{\prime \prime}$ and $z^{*}$, respectively. 
Corollary 3.4 Let $C$ be a nonempty, closed and convex subset of a real Hilbert space H. Let $T_{i}: C \times C \times C \rightarrow H$ be a relaxed $\left(\gamma_{i}, \delta_{i}\right)$-cocoercive and $\beta_{i}$-Lipschitz continuous mapping in the first variable and $g_{i}: C \rightarrow C$ be a relaxed $\left(\bar{\eta}_{i}, \bar{\rho}_{i}\right)$-cocoercive and $\bar{\lambda}_{i}$-Lipschitz continuous mapping for each $i=1,2,3$. Suppose that $\left(x^{\prime \prime}, y^{\prime \prime}, z^{\prime \prime}\right) \in C \times C \times C$ is a solution to the problem (1.8). Let $\left\{x_{n}\right\},\left\{y_{n}\right\}$ and $\left\{z_{n}\right\}$ be the sequences generated by Algorithm 2.3. Assume that the following conditions are satisfied:

(a) $\sum_{n=0}^{\infty} \alpha_{n}=\infty$;

(b) $0 \leq \theta_{6}, \theta_{9}<1$;

(c) $\theta_{1} \theta_{4} \theta_{7} \leq\left(1-\theta_{3}\right)\left(1-\theta_{6}\right)\left(1-\theta_{9}\right)$,

where

$$
\begin{aligned}
\theta_{1} & =\sqrt{1-2 s \delta_{1}+2 s \gamma_{1} \beta_{1}^{2}+s^{2} \beta_{1}^{2}}, & \theta_{3} & =\sqrt{1-2 \bar{\rho}_{1}+\bar{\lambda}_{1}^{2}+2 \bar{\eta}_{1} \bar{\lambda}_{1}^{2}}, \\
\theta_{4} & =\sqrt{1-2 t \delta_{2}+2 t \gamma_{2} \beta_{2}^{2}+t^{2} \beta_{2}^{2}}, & \theta_{6} & =\sqrt{1-2 \bar{\rho}_{2}+\bar{\lambda}_{2}^{2}+2 \bar{\eta}_{2} \bar{\lambda}_{2}^{2}},
\end{aligned}
$$

and

$$
\theta_{7}=\sqrt{1-2 r \delta_{3}+2 r \gamma_{3} \beta_{3}^{2}+r^{2} \beta_{3}^{2}}, \quad \theta_{9}=\sqrt{1-2 \bar{\rho}_{3}+\bar{\lambda}_{3}^{2}+2 \bar{\eta}_{3} \bar{\lambda}_{3}^{2}} .
$$

Then the sequences $\left\{x_{n}\right\},\left\{y_{n}\right\}$ and $\left\{z_{n}\right\}$ converge strongly to $x^{\prime \prime}, y^{\prime \prime}$ and $z^{\prime \prime}$, respectively.

Corollary 3.5 Let $C$ be a nonempty, closed and convex subset of a real Hilbert space H. Let $T_{i}: C \times C \times C \rightarrow H$ be a relaxed $\left(\gamma_{i}, \delta_{i}\right)$-cocoercive and $\beta_{i}$-Lipschitz continuous mapping in the first variable for each $i=1,2,3$. Suppose that $\left(x^{*}, y^{\prime \prime}, z^{*}\right) \in C \times C \times C$ is a solution to the problem (1.10). Let $\left\{x_{n}\right\},\left\{y_{n}\right\}$ and $\left\{z_{n}\right\}$ be the sequences generated by Algorithm 2.4. Assume that the following conditions are satisfied:

(a) $\sum_{n=0}^{\infty} \alpha_{n}=\infty$;

(b) $\theta_{1} \theta_{4} \theta_{7} \leq 1$,

where

$$
\theta_{1}=\sqrt{1-2 s \delta_{1}+2 s \gamma_{1} \beta_{1}^{2}+s^{2} \beta_{1}^{2}}, \quad \theta_{4}=\sqrt{1-2 t \delta_{2}+2 t \gamma_{2} \beta_{2}^{2}+t^{2} \beta_{2}^{2}},
$$

and

$$
\theta_{7}=\sqrt{1-2 r \delta_{3}+2 r \gamma_{3} \beta_{3}^{2}+r^{2} \beta_{3}^{2}} .
$$

Then the sequences $\left\{x_{n}\right\},\left\{y_{n}\right\}$ and $\left\{z_{n}\right\}$ converge strongly to $x^{\prime \prime}, y^{\prime \prime}$ and $z^{*}$, respectively.

Corollary 3.6 Let $C$ be a nonempty, closed and convex subset of a real Hilbert space H. Let $T_{i}: C \rightarrow H$ be a relaxed $\left(\gamma_{i}, \delta_{i}\right)$-cocoercive and $\beta_{i}$-Lipschitz continuous mapping, $f_{i}: C \rightarrow H$ be a relaxed $\left(\eta_{i}, \rho_{i}\right)$-cocoercive and $\lambda_{i}$-Lipschitz continuous mapping and $g_{i}: C \rightarrow C$ be a relaxed $\left(\bar{\eta}_{i}, \bar{\rho}_{i}\right)$-cocoercive and $\bar{\lambda}_{i}$-Lipschitz continuous mapping for each $i=1,2,3$. Suppose that $\left(x^{*}, y^{*}, z^{*}\right) \in C \times C \times C$ is a solution to the problem (1.12). Let $\left\{x_{n}\right\},\left\{y_{n}\right\}$ and $\left\{z_{n}\right\}$ be the sequences generated by Algorithm 2.5. Assume that the following conditions are satisfied:

(a) $\sum_{n=0}^{\infty} \alpha_{n}=\infty$;

(b) $0 \leq \theta_{6}, \theta_{9}<1$;

(c) $\left(\theta_{1}+\theta_{2}\right)\left(\theta_{4}+\theta_{5}\right)\left(\theta_{7}+\theta_{8}\right) \leq\left(1-\theta_{3}\right)\left(1-\theta_{6}\right)\left(1-\theta_{9}\right)$, 
where

$$
\begin{aligned}
& \theta_{1}=\sqrt{1-2 s \delta_{1}+2 s \gamma_{1} \beta_{1}^{2}+s^{2} \beta_{1}^{2}}, \quad \theta_{2}=\sqrt{1-2 \rho_{1}+\lambda_{1}^{2}+2 \eta_{1} \lambda_{1}^{2}}, \\
& \theta_{3}=\sqrt{1-2 \bar{\rho}_{1}+\bar{\lambda}_{1}^{2}+2 \bar{\eta}_{1} \bar{\lambda}_{1}^{2}}, \quad \theta_{4}=\sqrt{1-2 t \delta_{2}+2 t \gamma_{2} \beta_{2}^{2}+t^{2} \beta_{2}^{2}}, \\
& \theta_{5}=\sqrt{1-2 \rho_{2}+\lambda_{2}^{2}+2 \eta_{2} \lambda_{2}^{2}}, \quad \theta_{6}=\sqrt{1-2 \bar{\rho}_{2}+\bar{\lambda}_{2}^{2}+2 \bar{\eta}_{2} \bar{\lambda}_{2}^{2}}, \\
& \theta_{7}=\sqrt{1-2 r \delta_{3}+2 r \gamma_{3} \beta_{3}^{2}+r^{2} \beta_{3}^{2}}, \quad \theta_{8}=\sqrt{1-2 \rho_{3}+\lambda_{3}^{2}+2 \eta_{3} \lambda_{3}^{2}},
\end{aligned}
$$

and

$$
\theta_{9}=\sqrt{1-2 \bar{\rho}_{3}+\bar{\lambda}_{3}^{2}+2 \bar{\eta}_{3} \bar{\lambda}_{3}^{2}}
$$

Then the sequences $\left\{x_{n}\right\},\left\{y_{n}\right\}$ and $\left\{z_{n}\right\}$ converge strongly to $x^{*}, y^{*}$ and $z^{*}$, respectively.

\section{Competing interests}

The author declares that they have no competing interests.

\section{Acknowledgements}

The author is grateful to the editors, Kejifazhan (NO. 112400430123), and Jichuheqianyanjishuyanjiu (NO. 112400430123), Henan.

Received: 17 October 2012 Accepted: 23 November 2012 Published: 28 December 2012

\section{References}

1. Chang, SS, Yao, JC, Kim, JK, Yang, L: Iterative approximation to convex feasibility problems in Banach space. Fixed Point Theory Appl. 2007, 046797 (2007)

2. Zegeye, $\mathrm{H}$, Shahzad, N: Strong convergence theorem for a common point of solution of variational inequality and fixed point problem. Adv. Fixed Point Theory 2, 374-397 (2012)

3. Cho, SY, Kang, SM: Approximation of common solutions of variational inequalities via strict pseudocontractions. Acta Math. Sci. 32, 1607-1618(2012)

4. Husain, S, Gupta, S: A resolvent operator technique for solving generalized system of nonlinear relaxed cocoercive mixed variational inequalities. Adv. Fixed Point Theory 2, 18-28 (2012)

5. Kim, JK, Cho, SY, Qin, X: Hybrid projection algorithms for generalized equilibrium problems and strictly pseudocontractive mappings. J. Inequal. Appl. 2010, Article ID 312602 (2010)

6. Ye, J, Huang, J: Strong convergence theorems for fixed point problems and generalized equilibrium problems of three relatively quasi-nonexpansive mappings in Banach spaces. J. Math. Comput. Sci. 1, 1-18 (2011)

7. Qing, Y, Kim, JK: Weak convergence of algorithms for asymptotically strict pseudocontractions in the intermediate sense and equilibrium problems. Fixed Point Theory Appl. 2012, 132 (2012)

8. Kang, SM, Cho, SY, Liu, Z: Convergence of iterative sequences for generalized equilibrium problems involving inverse-strongly monotone mappings. J. Inequal. Appl. 2010, Article ID 827082 (2010)

9. LV, S: Generalized systems of variational inclusions involving (A, $\eta$-)monotone mappings. Adv. Fixed Point Theory 1 , 1-14 (2011)

10. Cho, SY, Kang, SM: Approximation of fixed points of pseudocontraction semigroups based on a viscosity iterative process. Appl. Math. Lett. 24, 224-228 (2011)

11. Qin, $X$, Cho, YJ, Kang, SM: Convergence theorems of common elements for equilibrium problems and fixed point problems in Banach spaces. J. Comput. Appl. Math. 225, 20-30 (2009)

12. Abdel-Salam, HS, Al-Khaled, K: Variational iteration method for solving optimization problems. J. Math. Comput. Sci. 2, 1457-1497 (2012)

13. Liu, M, Chang, SS: A new iterative method for solving a system of general variational inequalities. Adv. Nonlinear Var. Inequal. 12, 51-60 (2009)

14. Qin, X, Cho, SY, Kang, SM: Strong convergence of shrinking projection methods for quasi- $\phi$-nonexpansive mappings and equilibrium problems. J. Comput. Appl. Math. 234, 750-760 (2010)

15. Kim, JK: Strong convergence theorems by hybrid projection methods for equilibrium problems and fixed point problems of the asymptotically quasi- $\boldsymbol{\phi}$-nonexpansive mappings. Fixed Point Theory Appl. 2011,10 (2011)

16. Huang, Z, Noor, MA: An explicit projection method for a system of nonlinear variational inequalities with different $(\gamma, r)$-cocoercive mappings. Appl. Math. Comput. 190, 356-361 (2007)

17. Chang, SS, Joseph Lee, HW, Chan, CK: Generalized system for relaxed cocoercive variational inequalities in Hilbert spaces. Appl. Math. Lett. 20, 329-334 (2007)

18. Noor, MA, Noor, Kl: Projection algorithms for solving a system of general variational inequalities. Nonlinear Anal. 70 2700-2706 (2009) 
19. Qin, X, Cho, SY, Kang, SM: On hybrid projection methods for asymptotically quasi- $\phi$-nonexpansive mappings. Appl. Math. Comput. 215, 3874-3883 (2010)

20. $\mathrm{LV}, \mathrm{S}, \mathrm{Wu}, \mathrm{C}$ : Convergence of iterative algorithms for a generalized variational inequality and a nonexpansive mapping. Eng. Math. Lett. 1, 44-57 (2012)

21. Noor, MA: General variational inequalities. Appl. Math. Lett. 1, 119-121 (1988)

22. Qin, X, Chang, SS, Cho, YJ: Iterative methods for generalized equilibrium problems and fixed point problems with applications. Nonlinear Anal. 11, 2963-2972 (2010)

23. Zhang, M: Iterative algorithms for common elements in fixed point sets and zero point sets with applications. Fixed Point Theory Appl. 2012, 21 (2012)

24. Verma, RU: Generalized system for relaxed cocoercive variational inequalities and its projection methods. J. Optim. Theory Appl. 121, 203-210 (2004)

25. Verma, RU: General convergence analysis for two-step projection methods and application to variational problems. Appl. Math. Lett. 18, 1286-1292 (2005)

26. Verma, RU: Projection methods, algorithms, and a new system of nonlinear variational inequalities. Comput. Math. Appl. 41, 1025-1031 (2001)

27. Stampacchia, G: Formes bilineaires coercivites sur les ensembles convexes. C. R. Acad. Sci. Paris 258, 4413-4416 (1964)

28. Dunn, JC: Convexity, monotonicity and gradient processes in Hilbert spaces. J. Math. Anal. Appl. 53, 145-158 (1976)

29. Reich, S: Constructive techniques for accretive and monotone operators, applied nonlinear analysis. In: Proceedings of the Third International Conference University of Texas, Arlington, TX, 1978, pp. 335-345. Academic Press, New York (1979)

doi:10.1186/1687-1812-2012-232

Cite this article as: Zhang: Iterative algorithms for a system of generalized variational inequalities in Hilbert spaces.

Fixed Point Theory and Applications 2012 2012:232.

\section{Submit your manuscript to a SpringerOpen ${ }^{\circ}$ journal and benefit from:}

- Convenient online submission

- Rigorous peer review

- Immediate publication on acceptance

- Open access: articles freely available online

- High visibility within the field

- Retaining the copyright to your article

Submit your next manuscript at $\boldsymbol{s p r i n g e r o p e n . c o m ~}$ 